\title{
Characteristics of the spontaneous deposition of Sn in polycrystalline Pt
}

\author{
Javier E. Rodríguez Yáñez \\ Laboratorio de Química Marina, Universidad Nacional, Heredia, Costa Rica
}

Received 18 April 2012; accepted 30 April 2012

\begin{abstract}
Spontaneous deposition of tin $(\mathrm{Sn})$ in polycrystalline platinum $(\mathrm{Pt} p c)$ from a sulfuric acid solution of tin salts, was evaluated by conventional electrochemical techniques, to determine its characteristics.

Tin was deposited from a solution of $\mathrm{SnCl}_{2}$ or $\mathrm{SnSO}_{4}$ in sulfuric acid $1 \mathrm{M}$.

The degree of surface coating by $\mathrm{Sn}$ was calculated by evaluating the region of hydrogen electrosorption through voltammograms performed using solutions containing only the supporting electrolyte, and the supporting electrolyte containing the $\mathrm{Sn}$ salt, respectively.

The adsorption behavior of tin and oxidation have been studied by the results obtained with platinum electrodes and electrodes of platinum/tin, considering as comparative parameter the adsorption efficiency with respect to the area free of platinum.
\end{abstract}

Keywords: tin, platinum, spontaneous, electrocatalysis, voltammetry.

\section{Introducción}

La modificación de superficies metálicas por deposición de otros metales es una de las técnicas más usuales para la generación y evaluación de nuevos catalizadores.

La química verde plantea actualmente una tendencia a la minimización de las energías necesarias para la generación de un nuevo producto, así como la disminución de la toxicidad en su producción. En este sentido, las técnicas electroquímicas son una de las metodologías empleadas, las cuales permiten optimizar la generación de estas superficies metálicas compuestas [1].

Dentro de las técnicas más usuales de obtención de estas superficies por vía electroquímica se encuentra i) el depósito de metales a potenciales positivos con respecto al potencial de la cupla metálica $\mathrm{E}_{\mathrm{Mz}+\mathrm{M}}^{0}$, llamado depósito a subpotenciales (under potential deposition o upd); ii) la deposición a potenciales

\footnotetext{
* Corresponding author. E-mail: urutico@gmail.com
} 
más negativos que el de esa cupla metálica o deposición a sobrepotenciales (over potential deposition u $o p d$ ); iii) técnicas mixtas que combinan ambos potenciales en sistemas cíclicos, derivados del concepto de estabilización dinámica, u otras técnicas complejas [2-8].

Hace algunos años surgió con algunos autores el concepto de deposición espontánea, esto es, sin aplicación de corriente o potencial. En este sistema la deposición depende de las condiciones de la superficie, del medio electrolítico y de la naturaleza del metal a depositar [2-5].

La existencia de diferentes métodos de deposición, hace particularmente atractiva la consideración de la deposición espontánea (esp), en virtud de su facilidad, rapidez y relativa estabilidad [9-14].

Dependiendo de la técnica, se logran en general cubrimientos superficiales parciales, con estabilidad y reactividad variable y compleja [15-18].

Otra consideración según la situación específica, es la relativa selectividad por algunos tipos de planos cristalinos en la deposición, según el metal a depositar y el metal base, lo cual condiciona sus posibles capacidades catalíticas [19-22].

En el caso del Sn las características del depósito formado en las distintas técnicas son discutidas ampliamente, siendo en general descriptas para el estaño como una mezcla de $\mathrm{Sn}, \mathrm{SnO}, \mathrm{SnO}_{2}, \mathrm{Sn}(\mathrm{OH})_{2}$, principalmente [23-38].

En general, se reporta que los depósitos de $\mathrm{Sn}$ se producen en islas sobre estructuras cristalinas de Pt con orientaciones (110), proponiendo que su actividad está asociada a la situación de carga parcial o a la posibilidad de dismutar desde su estado como $\mathrm{Sn}^{2+}[8,39,40]$.

\section{Metodología \\ Electrodos}

Los experimentos fueron realizados usando alambres de platino policristalino (pc) de 0,5 mm de diámetro y 99,999\% de pureza (Good Fellow Co.) como electrodos de trabajo.

Como contra electrodo se utilizó un electrodo de platino de gran área, más de 10 $\mathrm{cm}^{2}$ y como electrodo de referencia un electrodo normal de hidrógeno $(E N H)$ generado con la disolución de trabajo. Todas las medidas se refieren al ENH.

El área real del electrodo de trabajo fue determinada por integración matemática del área de adsorción de hidrógeno. Los resultados son expresados en función de esta área disponible.

\section{Disoluciones}

El electrolito soporte utilizado fue $\mathrm{H}_{2} \mathrm{SO}_{4} 1 \mathrm{~mol}^{*} \mathrm{~L}^{-1}$, preparado con un ácido Baker (97,8\% ACS), diluido con agua de calidad Millipore MilliQ plus (resistividad 18,2 $\mathrm{M} \Omega \mathrm{cm}$ ).

La disolución para la deposición $0,1 \mathrm{mmol}^{*} \mathrm{~L}^{-1}$ en estaño, fue preparada a partir de $\mathrm{SnSO}_{4}$ (Sigma Aldrich 99,5\%) o $\mathrm{SnCl}_{2} \cdot 2 \mathrm{H}_{2} \mathrm{O}$ (Sigma Aldrich 99,9\%) en ácido sulfúrico $1 \mathrm{~mol}^{*} \mathrm{~L}^{-1}$. 


\section{Métodos de deposición}

A fin de evaluar los efectos del Sn en $\mathrm{Pt} p c$, se realizaron las deposiciones del mismo, como se indica en la literatura $[10,11,13,40]$, según la siguiente metodología:

Deposición espontánea de estaño por inmersión del platino en una disolución del mismo a circuito abierto o potencial libre de disolución.

Todas las deposiciones fueron realizadas desoxigenando las disoluciones con nitrógeno de alta pureza (Air Lyquide 5.0).

Los depósitos de estaño obtenidos fueron estabilizados por voltamperometría cíclica, entre $0 \mathrm{~V}$ y $0,6 \mathrm{~V}$, a una velocidad de barrido de $0,1 \mathrm{~V} \mathrm{~s}^{-1}$, en ácido sulfúrico $1 \mathrm{~mol}^{*} \mathrm{~L}^{-1}$.

\section{Cálculos de cubrimiento}

El valor de cubrimiento superficial $(\theta)$ se define como [34]:

$$
\theta=\left(\mathrm{Q}_{\mathrm{H}}-\mathrm{Q}_{\mathrm{H}, \mathrm{Ad}}\right) / \mathrm{Q}_{\mathrm{H}}
$$

donde $\mathrm{Q}_{\mathrm{H}}$ representa la carga de hidrógeno adsorbido por el electrodo de platino antes de la deposición y $\mathrm{Q}_{\mathrm{HAd}}$ representa la carga después de la deposición, ambas en ácido sulfúrico $1 \mathrm{~mol}^{*} \mathrm{~L}^{-1}$.

Se considera para los cálculos de Q el área anódica generada entre 0,05 V y 0,4 V vs. ENH.

Para la deposición espontánea el ajuste del cubrimiento se realiza por deposiciones repetidas con estabilizaciones intermedias. Por ejemplo, para 2 minutos de deposición espontánea se obtiene alrededor de un $15 \%$ a $20 \%$, con una solución preparada en el día.

La disolución para deposición espontánea presenta degradación con el tiempo, decreciendo consecuentemente su efectividad, por lo cual el tiempo máximo recomendado de uso es una semana $[35,41,42]$.

\section{Resultados y discusión \\ La deposición de Sn en Pt}

La superficie de platino fue modificada por deposiciones de estaño, con niveles de cubrimiento $\theta$ del orden de $10 \%$ a 50\%. Luego de modificada la superficie se realizó su estabilización.

El perfil voltamperométrico del $\mathrm{Pt} p c$ a $0,1 \mathrm{~V}^{*} \mathrm{~s}^{-1}$ antes y después de la deposición de Sn puede observarse en la Fig. 1. Las voltamperometrías fueron corridas entre $0,05 \mathrm{~V}$ y $0,6 \mathrm{~V}$ a fin de prevenir la desorción oxidativa del estaño depositado. Se utilizó esa región de potencial porque se ha reportado en varios artículos que el $\mathrm{Sn}$ depositado no se oxida pasando a disolución hasta potenciales superiores a $0,8 \mathrm{~V}[36]$.

La comparación entre los perfiles antes y después de la deposición evidencian la inhibición parcial de la adsorción de hidrógeno por parte del estaño depositado, así como el engrosamiento de la doble capa. Varios autores ya han indicado esta situación, marcando incluso preferencias por regiones cristalinas 
correspondientes al primer pico de adsorción de hidrógeno y la dependencia del efecto con el grado de cubrimiento [34,42,44].

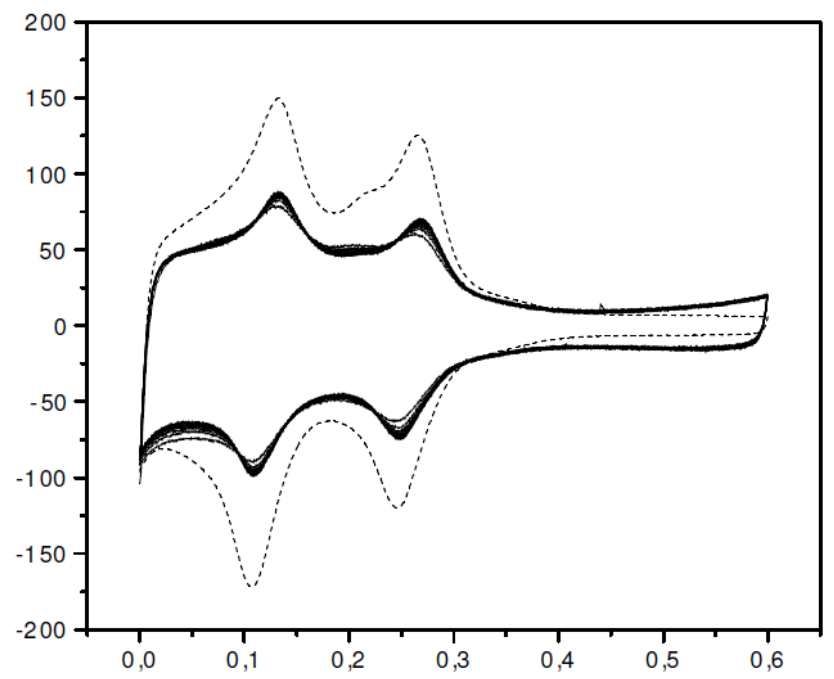

Figura 1. Voltamperometría cíclica entre $0,05 \mathrm{~V}$ y $0,6 \mathrm{~V}$ a $0,1 \mathrm{~V}^{*} \mathrm{~s}^{-1}$, (10 ciclos). Estabilización de los depósitos de $\mathrm{Sn}$ en Pt por ciclado en la región de upd. Depósito Sn espontáneo $32 \%$ (-), Pt limpio (---).

Paralelamente se observó que se producía una estabilización de los recubrimientos generados al realizar la voltamperometria cíclica para comprobar el porcentaje de recubrimiento, generando un reordenamiento parcial de los sitios de adsorción, lo cual puede verse en la estabilización de los picos de la región de adsorción de hidrógeno en la Fig. 1.

Esta estabilización permite una mejor repetibilidad del proceso de deposición espontánea, lográndose de ese modo realizar varias deposiciones sobre la superficie, con valores de cubrimiento similares, según las condiciones de deposición utilizadas y llegando a obtener cubrimientos mayores que $50 \%$.

\section{Tiempo de deposición}

Como primera consideración se realizó la deposición con la misma disolución, variando el tiempo de deposición y el tiempo de vida de la solución.

Se observó que el tiempo de deposición es relativamente rápido, obteniendo porcentajes de cubrimientos similares y estables del orden del 15 al $20 \%$, para tiempos de unos 2 minutos.

En la Fig. 2, puede verse el crecimiento de la deposición espontánea con el tiempo, para una disolución de una semana. En ella se aprecia el crecimiento con el tiempo de la deposición espontánea, llegando a una meseta cerca de los 10 min. Esto plantea una relación de tipo logarítmica entre la adsorción y el tiempo de interacción, del tipo:

$$
\theta_{\text {Sn }}(\%)=2,109 \ln (\mathrm{t}(\mathrm{s}))+13,77 \quad \mathrm{R}^{2}=0,997
$$

Por otro lado la edad de la disolución mostró ser una variable importante, obteniéndose una dependencia con la misma en la deposición espontánea que se 
producía. Los depósitos pasaban de valores de más del 30\% para disoluciones recién preparadas (freshly prepared), a valores del orden de $20 \%$ para disoluciones de una semana de preparadas, con tiempos de deposición de unos 10 min, lo cual puede apreciarse en la Fig. 3 [13,23,45,46].

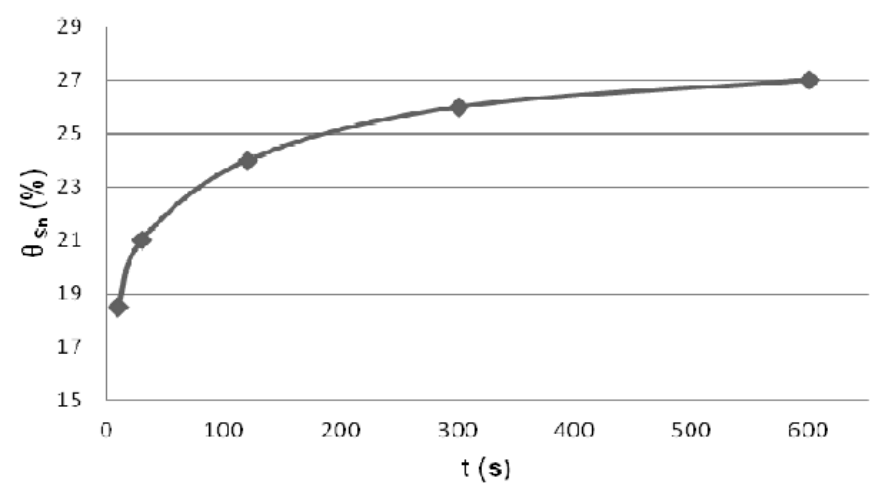

Figura 2. Variación del cubrimiento $\left(\theta_{\mathrm{Sn}}\right)$ con el tiempo de deposición espontánea sobre un electrodo de $\mathrm{Pt} p c$. Disolución de $\mathrm{SnSO}_{4}$ 0,1 $\mathrm{mM}$ en ácido sulfúrico $1 \mathrm{M}$, antigüedad de una semana.

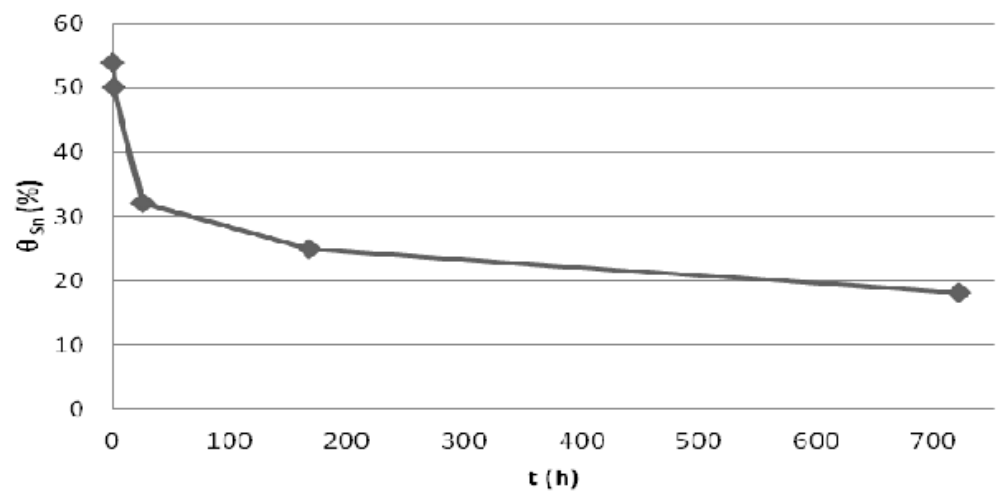

Figura 3. Variación del cubrimiento $\left(\theta_{\mathrm{Sn}}\right)$ por deposición espontánea en un electrodo de Pt $p c$, con la edad de la solución de $\mathrm{SnCl}_{2} 0,1 \mathrm{mM}$ en ácido sulfúrico $1 \mathrm{M}$, para un tiempo de deposición de 10 minutos.

El decaimiento se puede aproximar para los valores luego de $1 \mathrm{~h}$, a una curva logarítmica, según la ecuación:

$$
\theta_{\text {Sn }}(\%)=-4,82 \ln (\mathrm{t}(\mathrm{h}))+49,23 \quad \mathrm{R}^{2}=0,993
$$

De este comportamiento y considerando una adsorción espontánea de $\mathrm{Sn}$, basado en una isoterma de Frumkin del tipo:

$$
\ln (1-\theta / \theta)=(\mathrm{r} / \mathrm{RT}) \theta-\ln \mathrm{a}+\left(\Delta \mathrm{G}^{\mathrm{o}} \text { ads } / \mathrm{RT}\right)
$$

La misma presenta valores de interacción lateral de repulsión $\mathrm{r}=-14 \mathrm{~kJ} / \mathrm{mol}$ y un $\Delta \mathrm{G}^{\mathrm{o}}$ ads $=-26 \mathrm{~kJ} / \mathrm{mol}$, asimilable a una adsorción física dado que es menor que $-40 \mathrm{~kJ} / \mathrm{mol}[47]$.

\section{Efecto del contraión}

La influencia del contraión asociado al estaño, se probó midiendo los niveles de adsorción para una disolución de una semana, con $\mathrm{SnSO}_{4}$ o $\mathrm{SnCl}_{2}$. 
El proceso indicó que la reacción es débilmente dependiente del contraión, debido a que en ambos casos se midieron adsorciones similares, aunque las soluciones de sulfato de estaño presentaron adsorciones ligeramente mayores, pero no significativas.

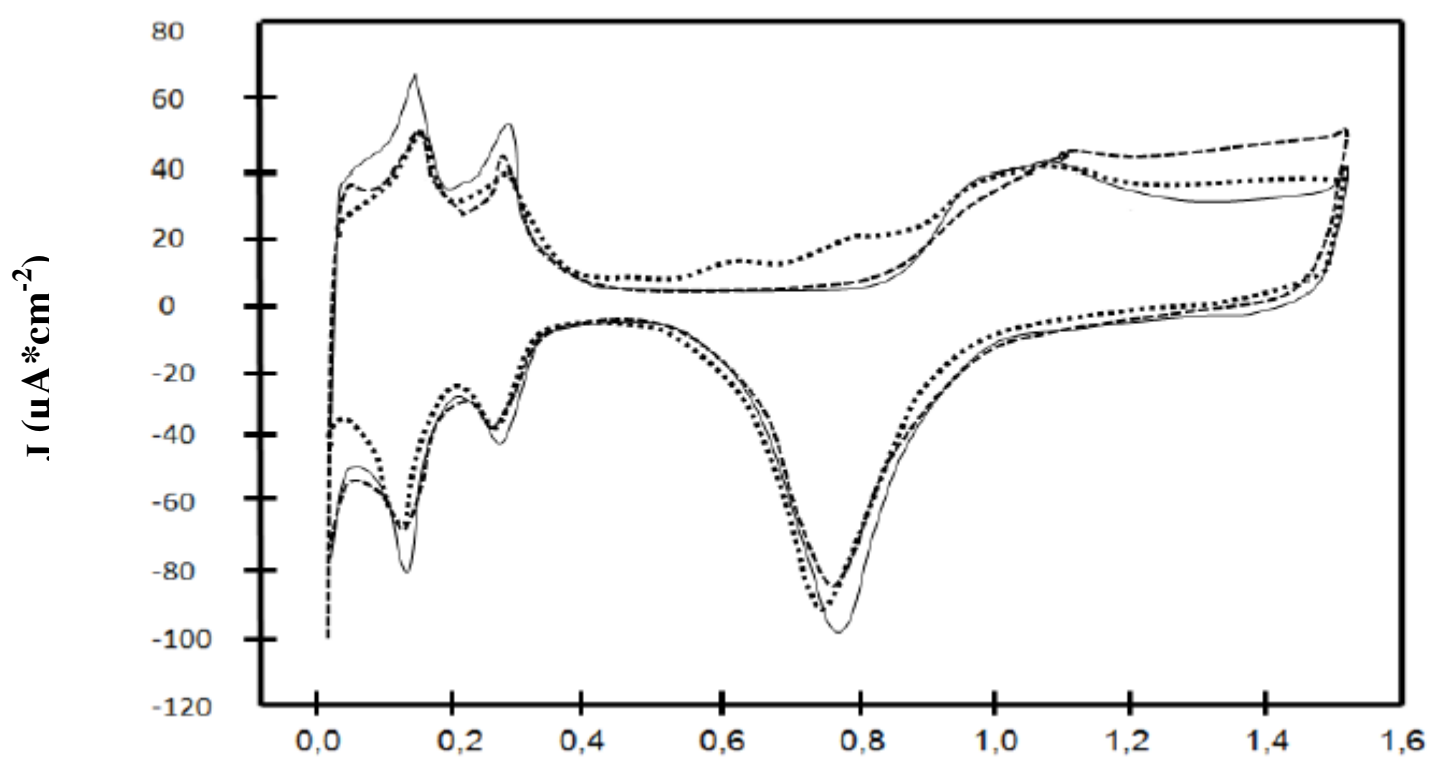

\section{E vs. ENH (V)}

Figura 4. Voltamperometría cíclica de la oxidación de Sn espontáneo sobre Pt $p c$ : Pt/Sn espontáneo 22\% freshly prepared (---) sin estabilizar, Pt/Sn espontáneo $27 \%$ envejecido multi depósito ('..) estabilizado, Pt pc en ácido sulfúrico 1M (-).

\section{Estabilidad de la deposición espontanea}

La estabilidad de los depósitos generados se visualizó inicialmente realizando voltamperometrías cíclicas en la región de potenciales de estabilidad termodinámica del Pt en ácido sulfúrico 1 M. Las mismas fueran realizadas considerando dos situaciones con el objetivo de obtener altos cubrimientos similares: i) la utilización de soluciones recién preparadas sin estabilización y ii) la utilización de deposiciones espontáneas múltiples estabilizadas. Estas situaciones se comparan con el electrodo de Pt $p c$ y se muestran en la Fig. 4.

Se aprecia picos de oxidación a potenciales superiores a $0,6 \mathrm{~V}$ para un depósito recién preparado sin estabilización, mientras que para deposición en etapas múltiples estabilizadas, los picos son leves o mínimos, dándose una deformación de la región de óxidos de Pt [10,11,25-34].

Asimismo se realizaron cronopotenciometrías de los depósitos frente a la solución de deposición de $\mathrm{Sn}^{2+} 2 \mu \mathrm{M}$ en ácido sulfúrico $1 \mathrm{M}$. Se plantearon tres situaciones básicas: i) el electrodo de Pt $p c$ limpio; ii) un electrodo de $\mathrm{Pt} / \mathrm{Sn}$ con un depósito de espontáneo sin estabilizar y iii) un electrodo de $\mathrm{Pt} / \mathrm{Sn}$ con un depósito de espontáneo estabilizado, que se muestran en la Fig. 5. 
Observando los resultados se deduce que el proceso de adsorción superficial sobre Pt $p c$ limpio tiene un potencial inicial similar al del $p z c$, que decae con el tiempo hasta estabilizarse a unos $100 \mathrm{~s}$, alcanzando una meseta de potencial $[48,49]$.

Por otro lado la existencia de un depósito espontáneo previo sin estabilizar, genera un decaimiento continuo del potencial. Pero cuando el mismo es estabilizado previamente, el valor de potencial es casi constante.

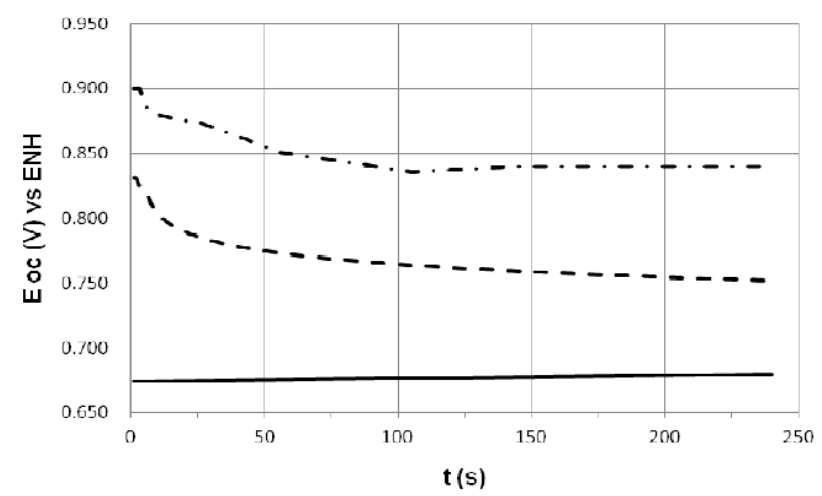

Figura 5. Cronopotenciometría de $\mathrm{Pt} p c$ en solución de $\mathrm{SnSO}_{4} 0,1 \mathrm{mM}$ en ácido sulfúrico $1 \mathrm{M}$ para deposición espontánea $\left(\left(^{-}\right)\right.$. Electrodo de $\mathrm{Pt} / \mathrm{Sn}$ espontáneo, sin estabilizar $(\theta=18 \%)(--)$ y $\mathrm{Pt} / \mathrm{Sn}$ espontáneo $(\theta=20 \%)$ estabilizado $(-)$ en ácido sulfúrico $1 \mathrm{M}$.

\section{Discusión de resultados}

Los experimentos cronopotenciométricos relacionados con la deposición indican que el proceso modifica la carga superficial, estabilizando el potencial de reposo en valores unos $50 \mathrm{mV}$ menores. Esto puede asociarse a que la adsorción primaria del $\mathrm{Sn}^{2+}$ sobre la superficie de $\mathrm{Pt} p c$, que de acuerdo a los resultados de la isoterma de adsorción es de tipo físico, confirmado por los valores de $\Delta \mathrm{G}_{\mathrm{ads}}$, conduciendo a la modificación del potencial del sistema superficial.

La estabilización posterior por ciclado potenciodinámico en la región de upd $(0,05$ a 0,6 V), permite obtener una adsorción más fuerte que la inicial, debido a que se está en una región de adsorción química. Asimismo, se reordenan los sitios de adsorción como puede observarse a través de la modificación de los picos de adsorción de $\mathrm{H}$.

El ciclado potenciodinámico mejora la estabilización del depósito frente a la oxidación del mismo al aumentar el potencial y lleva el potencial de reposo a 0,6 V valores ligeramente superior al límite de la región de upd.

De acuerdo a la bibliografía, los picos de oxidación se pueden asociar a las oxidaciones sucesivas de $\mathrm{Sn}$, sus óxidos o hidróxidos superficiales adsorbidos [23-38].

La existencia de depósitos previos (estabilizados o no) presenta potenciales de circuito abierto menores y una superficie modificada donde el Sn tiende más fácilmente a adsorberse.

También se puede ver que las deposiciones con bajos recubrimientos superficiales $(\theta)$, realizados sucesivamente y estabilizados cada vez, mejoran el 
comportamiento final del depósito de Sn frente a la oxidación. Este hecho está posiblemente asociado a la formación de islas de $\mathrm{Sn}$ sobre la superficie de $\mathrm{Pt}$ $[13,34,35]$.

El límite obtenido de un $60 \%$ de cubrimiento puede ser asociado a los niveles de repulsión encontrados para la adsorción física inicial.

El aumento de la edad de la disolución empleada para la deposición de Sn genera depósitos más estables, no obstante, presentan menores porcentajes de recubrimiento superficial $(\theta)$.

A concentraciones tan diluidas como las empleadas en este trabajo, el efecto del contraión no es significativo respecto al del medio.

\title{
Agradecimiento
}

Se agradece al Ministerio de Ciencia y Tecnología de Costa Rica, por el apoyo brindado al autor de este trabajo como becario de ese Ministerio.

\section{Características de la Deposición Espontánea de Sn en Pt policristalino}

\author{
Javier E. Rodríguez Yáñez
}

\section{Resumen}

La deposición espontánea de estaño $(\mathrm{Sn})$ en platino policristalino $(\mathrm{Pt} p c)$ desde una disolución acuosa de ácido sulfúrico conteniendo sales de estaño, se evaluó mediante el empleo de técnicas electroquímicas convencionales.

El estaño fue depositado desde una disolución de $\mathrm{SnCl}_{2}$ o $\mathrm{SnSO}_{4}$ en ácido sulfúrico 1 M.

El grado de recubrimiento de la superficie por Sn se calculó evaluando la región de electrosorción de hidrógeno en voltamperogramas realizados empleando soluciones conteniendo solamente el electrolito soporte y otros conteniendo el electrolito soporte y la sal de Sn.

El comportamiento de la adsorción de estaño y su oxidación fueron estudiados a través de los resultados obtenidos con electrodos de platino y electrodos de platino/estaño, considerando como parámetro comparativo la eficiencia de adsorción con respecto al área libre de platino.

Palabras clave: estaño, platino, espontáneo, electrocatálisis, voltamperometría.

\section{Referencias}

1. A.M. Peiro, Nuevas aportaciones al desarrollo de Metodologías en Química Verde, Tesis Doctoral, Universidad Autónoma de Barcelona, 2003.

2. O.A. Petrii, V.D. Kalinin, Rus. J. Electrochem. 35 (1999) 627.

3. M.M.P. Janssen, J. Moolhuysen, Electrochim. Acta 21 (1976) 869. 
4. J.C. Davies, B.E. Hayden, D.J. Pegg, M.E. Rendall, Surf. Sci. 496 (2002) 110.

5. P. Waszczuck, J. Solla-Guillon, H.S. Kim, Y.Y. Tong, V. Montiel, A. Aldaz, A. Wieckowski, J. Catal. 203 (2001) 1.

6. J. Mc. Breen, S. Mukerjee, J. Electrochem. Soc. 142 (1995) 3399.

7. H. von Schenck, E. Janin, O. Tjernberg, M. Svensson, M. Goethelid, Surf. Sci. 526 (2003) 184.

8. Yu. Morimoto, E.B. Yeager, J. Electroanal. Chem. 441 (1998) 77.

9. M. Watanabe, Y. Furuuchi and S. Motoo, J. Electroanal. Chem. 191 (1985) 367

10. G. Stalnionis, L. Tamasauskaite-Tamasiunaite, V. Pautieniene, Z. Jusys, J. Solid State Electrochem. 8 (2004) 892

11. G. Stalnionis, L. Tamasauskaite-Tamasiunaite, V. Pautieniene, Z. Jusys, J. Solid State Electrochem. 8 (2004) 900

12. Y. Ishikawa, M. Liao, C. R. Cabrera, Surf. Sci. 463 (2000) 66

13. S.H. Bonilla, C.F. Zinola, J. Rodríguez, V. Díaz, M. Ohanian, S. Martínez, B.F. Giannetti, J. Colloid. Int. Sci. 288 (2005) 377.

14. A.N. Frumkin, B.I. Podlovchenko, Ber. Akad. Wiss. USSR 150 (1963) 34.

15. S.J.C. Cleghorn, X. Ren, T.E. Springer, M.S. Wilson, C. Zawodinski, T.A. Zawodinski, S. Gottesfeld, Int. J. Hydrogen Energy 22 (1997) 1137.

16. B. Gurau, R. Viswanathan, R. Liu, T.J. Lafrenz, K.L. Ley, E.S. Smotkin, E. Reddington, A. Sapienza, B.C. Chan, T.E. Mallouk, S. Sarangapani, J. Phys. Chem. B 102 (1998) 9997.

17. H. Gerischer, D.M. Kolb, J.K. Sass, Adv. Phys. 27 (1978) 437.

18. K.A. Friedich, K.P.Geyzers, A.J. Dickinson, U. Stimming, Surf. Sci., 402404 (1998) 571.

19. C.K. Rhee, M. Wakisaka, Y.V. Tolmachev, C.M. Johnston, R. Haasch, K. Attenkofer, G.Q. Lu, H. You, A. Wieckowski, J. Electroanal. Chem. 554555 (2003) 367.

20. A. Crown, A. Wieckowski, Phys. Chem. Chem. Phys. 3 (2001) 3290.

21. T. Frelink, W .Visscher, J.A.R. van Veen, Langmuir 12 (1996) 3702.

22. R. Liu, H. Iddir, Q. Fan, G. Hou, A. Bo, K. L. Ley, E.S. Smotkin, Y.E. Sung, H. Kim, S. Thomas, A. Wieckowski, J. Phys. Chem. B 104 (2000) 3518.

23. E.V. Spinace, A.O. Neto, M. Linardi, J. Power Sources 129 (2004) 121.

24. F. Colom, M. González-Tejera, J. Appl. Electrochem. 24 (1994) 426.

25. A. Crown, I. de Moraes, A. Wieckowski, J. Electroanal. Chem. 500 (2001) 333.

26. F. Rodríguez, T. Morante, C.R. Cabrera, J. Electroanal. Chem. 571 (2004) 15.

27. Y. Li, B.E. Koel, Surf. Sci. 330 (1995) 193.

28. C. Gallis, B. Legrand, G. Traglia, Surf. Sci. 377-379 (1997) 1033.

29. M.C. Santos, L.O.S. Bulhoes, Electrochim. Acta 48 (2003) 2607.

30. F.P. Gorstema, J.W. Cobble, J. Am. Chem. Soc. 83 (1961) 4317.

31. M. Watanabe, S. Motoo, J. Electroanal. Chem. 60(1975) 267. 
32. M. Seruga, M. Metikos-Hukovic, T. Valla, M Mulin, H. Hoffschultz, K. Wandelt, J. Electroanal. Chem. 407 (1996) 83.

33. R. Holze, B. Bittins Cattaneo, Electrochem. Acta 33 (1988) 353

34. C.F. Zinola, J. Rodríguez, G. Obal, J. Appl. Electrochem. 31 (2001) 1293.

35. B.B. Cattaneo, T. Iwasita, J. Electroanal. Chem. 238 (1987) 151

36. J. Sobkowski, K. Franaszczuk and A. Piasecki, J. Electroanal. Chem. 196 (1985) 145

37. M.M.P. Janssen, J. Moolhuysen, J. Catal. 46 (1976) 289

38. E. Reddington, A. Sapienza, B. Gurau, R. Viswanathan, S. Sarangapani, E.S. Smotkin, T. Mallouk, Science 280 (1998) 1735.

39. N.M. Markovic, P.N. Ross, Surf. Sci. Rep. 45 (2002) 117.

40. H. Massong, S. Tillmann, T. Langkau, E. Abd El Meguid, H.Baltruschat, Electrochim. Acta 44 (1998) 1379.

41. V. Solis, T. Iwasita, A. Pavese, W. Vielstich, J. Electroanal. Chem. 255 (1988) 155.

42. S. Szabo, J. Electroanal. Chem. 172 (1984) 359.

43. I. Bakos, S. Szabo, Electrochim. Acta 46 (2001) 2507.

44. X.H. Xia, Electrochim. Acta 45 (1999) 1057.

45. T. Iwasita, H. Hoster, A. John-Anacker, W.F. Lin, W. Vielstich, Langmuir 16 (2000) 522.

46. P. Waszczuck, T.M. Barnard, C. Rice, R.I. Masel, A. Wieckowski, Electrochem. Comm. 4 (2002) 599.

47. P.W. Atkins, Química Física, Ed. Médica Panamericana, $8^{\mathrm{a}}$ edición, México, 2007.

48. C.M. Brett, A.M. Oliveira, Electrochemistry Principles, Methods, and Applications, Oxford University Press, New York, USA, 1993.

49. R. Gómez, V. Climent, J.M. Feliu, M.J. Weaver, J. Phys. Chem. B 104 (2000) 597. 